\title{
Perceptions and Experiences of Participants in the Internationally-Educated Engineers Qualification Pilot Program, University of Manitoba
}

\author{
Marcia Friesen, P.Eng. \\ Faculty of Engineering, University of Manitoba, Winnipeg, Manitoba, Canada, R3T 5V6 \\ Marcia_Friesen@UManitoba.ca
}

\begin{abstract}
With increasing immigration to Canada, governments are urging professional regulatory bodies to develop new licensing pathways to recognize foreign credentials of immigrant professionals more quickly and effectively. The Internationally-Educated Engineers Qualification Pilot Program was developed in response to such pressures. The objectives of this primarily qualitative research study were to document and understand the experiences and perceptions of the international engineering graduates in three program cohorts and to apply the findings to program development and evaluation. Data were obtained through focus groups and follow-up questionnaires administered to three cohorts. Findings indicated that program strengths, which are also aligned with program objectives, include its recognition as a licensing pathway, emphasis on cultural integration, and labour market access. Findings led to the development of a comprehensive support structure for participants. Keywords: international engineering graduate; foreign credentials recognition; qualitative research.
\end{abstract}

\section{Introduction}

When immigrants with non-Canadian engineering credentials arrive in Canada, they are often surprised to learn that, by law, they must be registered with a provincial engineering association (regulatory body) in order to practice engineering in Canada. Regulatory bodies have always provided licensing pathways for international engineering graduates (IEGs), often in the form of a set of assigned Confirmatory Exams. Governments are urging professional regulatory bodies to develop alternative licensing pathways that integrate IEGs more quickly and effectively while maintaining standards for public safety. The Canadian Council of Professional Engineers (CCPE) has demonstrated leadership among professions by engaging engineering regulatory bodies and industry nationally in the multi-phase From Consideration to Integration project [1].
Within the engineering profession, IEGs confirm the need for new licensing pathways, citing difficulties in foreign credentials recognition (FCR) and gaining Canadian engineering experience as the two primary obstacles to full labour market participation [1,2]. Engineering employers concur that the most important factors influencing IEGs' level of employment are prior related Canadian experience, communication skills, and professional licensure [1].

In response to these pressures, the InternationallyEducated Engineers Qualification Pilot Program (IEEQ) at the University of Manitoba was developed in 2003 and recognized by the Association of Professional Engineers and Geoscientists of Manitoba (APEGM) as an acceptable licensing pathway for IEGs, leading to Engineer-inTraining (EIT) registration in Manitoba. IEEQ is a 12month program comprised of eight months of senior-level engineering courses to confirm technical background, and a four month co-op term to gain Canadian engineering experience. IEEQ also includes cultural training via a mandatory core course Practicing Professional Engineering in Manitoba (PPEM) and individualized English language support.

IEEQ is well-positioned relative to immigration, economic, and regulatory realities. In Manitoba, annual immigration has increased from 3500 to 10,000 immigrants between 1999 and 2007, with a projected increase to 20,000 immigrants by 2017 [3,4]. Nationally, immigration is a powerful force in the Canadian economy. Immigrants made up $70 \%$ of labour force growth in the 1990s and are expected to make up 100\% of labour force growth by the year 2011 [1,5]. In the regulatory context, a Professional Engineer (P.Eng.) license is a legal requirement to practice professional engineering and a necessary credential for career advancement.

Universities lack the mandate and history to facilitate licensure for immigrant professionals, as licensing is a provincial responsibility delegated through legislation to regulatory bodies. The knowledge base for universitybased FCR programs (in all professions) is currently limited to identifying FCR initiatives and capacity at Canadian universities [6]. IEEQ's function as a formal 
FCR pathway, combined with multiple partnerships between the university, engineering regulator, government, and engineering industry make it unique in Canada. Although similar initiatives exist in other professions, the lack of parallel programs in engineering with which to compare frameworks for delivery, assessment and evaluation, participants' experiences, and program outcomes have led to these frameworks being selfgenerated within IEEQ $[7,8]$.

This exploratory study was designed to address this void in the academic engineering literature. The primary objective was to document and understand the experiences and perceptions of the first three IEEQ cohorts, in order to build a full picture of their participation in IEEQ. The secondary objective was to apply findings toward program development (assessment) and to qualitatively compare findings to program objectives (evaluation).

\section{Methodology}

The methodology used to document and understand the experiences and perceptions of the first three IEEQ cohorts (referred to as IEEQ1, IEEQ2, and IEEQ3, respectively) was primarily qualitative, combined with several quantitative descriptive measures. Qualitative methods allow inquiry into a social condition or experience, based on building a holistic picture, reported textually, and analyzed inductively. Qualitative methods engage small numbers of participants and are appropriate when desired findings include description, interpretation, and deep understanding of a phenomenon [9].

Data collection consisted of one focus group interview and two follow-up questionnaires with each of the first three IEEQ cohorts, with all measures relying on voluntary participation. The focus groups, timed for the end of the coursework, were designed following established guidelines for focus group interviews [10] to explore participants' experiences in the academic portion of IEEQ, perceptions of program strengths, weaknesses, and recommendations for changes. A semi-structured interview protocol guided data collection, and sessions typically lasted 90-120 minutes. All sessions were moderated by an experienced third party. The moderator took notes during the sessions which were later transcribed, returned to the participants for checking, and then submitted to the researcher for analysis. A full focus group protocol is available from the author.

The follow-up questionnaires were administered by regular mail to participants at nine and 24 months after program completion. The questionnaires explored participants' perceptions of and experiences in the co-op work term and career development post-IEEQ, including the nature of their work, strengths and challenges of the work term, and the impact of academic preparation. A combination of qualitative questions and quantitative career development descriptors were used. To ensure confidentiality, participants did not include identifying information on their responses, which were compiled by a third party and a summary provided to the researcher. Full questionnaire protocols are available from the author.

Qualitative data analysis consisted of data coding using the constant comparative method [11] in which data are examined for patterns, themes, and negative cases. Quantitative descriptors are reported by noting ranges and means when appropriate. The protocol was approved by the university's Research Ethics Board prior to implementation. Participation and response rates in the focus group and follow-up questionnaires are shown in Table 1.

Table 1. Timing, participation, and response rates in data collection events

\begin{tabular}{|c|c|c|c|}
\hline $\begin{array}{l}\text { Cohort } \\
(\mathrm{n}=\text { cohort size } \\
\text { that } \\
\text { successfully } \\
\text { completed } \\
\text { IEEQ) }\end{array}$ & $\begin{array}{l}\text { Focus group } \\
\text { ( } n=\text { number of } \\
\text { participants) }\end{array}$ & $\begin{array}{l}\text { Nine-month follow-up } \\
\text { question-naire } \\
\text { ( } \mathrm{n}=\text { number of } \\
\text { responses) }\end{array}$ & $\begin{array}{l}\text { 24-month follow-up } \\
\text { question-naire } \\
\text { (n=number of responses) }\end{array}$ \\
\hline IEEQ1 (n=5) & $\mathrm{n}=5$ (March 2004) & n=2 (June 2005) & n=4 (Sept 2006) \\
\hline IEEQ2 ( $(n=13)$ & n=9 (May 2005) & n=10 (June 2006) & Pending (Sept 2007) \\
\hline IEEQ3 $(n=8)$ & $\mathrm{n}=3$ (May 2006) & Pending (June 2007) & Pending (Sept 2008) \\
\hline
\end{tabular}

\section{Findings}

The primary objective of this study was to document and understand participants' experiences and perceptions in IEEQ. These findings should be interpreted as preliminary, based on the completed data collection events
(Table 1). Findings are contextualized to cohorts IEEQ1 through IEEQ3, and as with all qualitative research, findings are not automatically generalizable to all IEGs. 


\section{Participant profiles}

IEEQ participants typically ranged in age from late 20s to mid-40s. Most were men, had spouses and children, and had immigrated to Canada within the two years prior to beginning IEEQ. The top three countries / regions of origin were South Asia, Argentina, and Eastern Europe. The most common engineering disciplines were mechanical, electrical, and civil engineering. Table 2 summarizes cohort sizes, completion rates, and post-IEEQ licensing status.

IEEQ participants' perceptions of and experiences in IEEQ academic requirements, co-op work terms, and post-IEEQ career development

The focus group and follow-up questionnaires asked participants to reflect on their experiences during IEEQ, including the academic portion and the co-op work term. The 24-month follow-up questionnaire also asked participants to characterize their career development since completing IEEQ, within pre-defined parameters. Key findings are summarized in Tables 3 and 4.

All participants employed at the nine- and 24-month follow-up reported doing what they wanted to be doing at that time, and that their employment was related to their co-op employment in some way. At the 24-month followup, three of four respondents believed they would not have been in the same position in their career in Canada without having attended IEEQ. The findings reveal career development gains for former IEEQ participants in the nature of employment, salaries, and progress in meeting the requirements of the EIT and P.Eng. licensing stages with APEGM.

Table 2. Participation data and licensing status for IEEQ cohorts

\begin{tabular}{|c|c|c|c|c|c|}
\hline Cohort & Entered IEEQ & $\begin{array}{l}\text { Completed } \\
\text { IEEQ }\end{array}$ & Not completed & $\begin{array}{c}\text { Registered as } \\
\text { EIT }^{1} \\
\end{array}$ & $\begin{array}{l}\text { Registered as } \\
\text { P.Eng. }\end{array}$ \\
\hline IEEQ1 & 7 & 5 & 2 - voluntary withdrawal & 2 & 3 \\
\hline IEEQ2 & 14 & 13 & 1 - exited by IEEQ & 9 & 4 \\
\hline IEEQ3 & 9 & 7 & $\begin{array}{l}1 \text { - completion pending } \\
1 \text { - exited by IEEQ }\end{array}$ & 7 & $\begin{array}{c}\text { (not yet } \\
\text { possible) }\end{array}$ \\
\hline Totals: & 30 & $\begin{array}{l}25 \text {, plus } 1 \\
\text { pending }\end{array}$ & $\begin{array}{c}\text { 4- voluntary } \\
\text { withdrawal or exited }\end{array}$ & 18 & 7 \\
\hline
\end{tabular}

\section{Discussion}

The secondary objective of this study was to apply the findings toward ongoing program development (program assessment) and to qualitatively compare participant outcomes to program objectives (program evaluation).

\section{Assessment objective: ongoing program development}

At program inception, IEEQ was conceptualized as a licensing pathway recognized by APEGM, comprised of eight months of senior-level courses, a mandatory course PPEM, and a four-month co-op term. Over time, IEEQ added a participant support structure modeled on Canadian Access programs, which deliver student support along three pillars: academic, social, and financial [12]. Supports are delivered directly within IEEQ, combined with appropriate liaison with campus and community services. The evolution was motivated by feedback from IEEQ1, whose perceptions and experiences reflected a lack of understanding of program expectations, a sense of isolation individually and as an IEEQ cohort within the
Faculty of Engineering, and burdens of heavy workload and financial cost. The current IEEQ framework exemplifies a 'difference' vs. a 'deficit' model of qualifications recognition. The program emphasizes the 'Canadianization' of technical and professional knowledge, skills, and attitudes, equally to addressing gaps or deficiencies in an IEG's background.

\section{Evaluation objective: comparison of outcomes to IEEQ objectives}

The objectives of IEEQ are to facilitate professional licensure and Canadian engineering work experience, in which the program (a) is a time-effective alternative to other licensing pathways; (b) offers a supportive community for participants; and (c) offers an opportunity for progressive transition and integration into the Canadian engineering profession. The program structure has been shown to successfully facilitate these objectives. IEEQ is recognized by APEGM as an appropriate and complete substitute to other licensing pathways, and IEEQ has facilitated Engineer-in-Training registration for all 
Table 3. Key findings on participants' experiences in the IEEQ program

\author{
Parameter \\ Characterization of experience in IEEQ: challenging in \\ two main areas: \\ 1. Adjusting to the lifestyle and routine of university studies \\ (unfamiliar system; second language); and, \\ 2. Coordinating studies with other commitments (family and \\ community, acculturation, employment).
}

\section{Illustrative Quote}

"after being out of university for many years it is difficult to come back to school... [and] added responsibilities impact the adjustment"

\section{Strengths \& Benefits of IEEQ - Reflections after the academic portion of IEEQ}

A better/preferable vehicle for re-entry into the engineering profession than Confirmatory Exams: more extensive and better support mechanisms in place; more time-effective; provides documented Canadian education.

Emphasis on cultural integration and understanding relative to Canadian culture and professional engineering: via the mandatory course PPEM, industry tours, exposure to professional engineers (professors, IEEQ staff, guest speakers, and engineers-in-residence), and immersion into an English-speaking environment.

Support in being part of an IEEQ cohort: (contact to staff and fellow IEEQ participants)

Program's ability to support varied goals: P.Eng. licensing, labour market entry, upgrading knowledge, and/or preparing for graduate studies.

\section{Experiences and Perceptions of the Co-op Work Term}

Majority of co-op assignments were self-assessed as engineering work (as per APEGM definition of acceptable engineering experience)

Acknowledged re-focusing of past experience / expertise into new industries in Canada

Self-reported strong match between co-op assignments and self-assessed capabilities, engineering background, and/or interests

Infrequent formal feedback; appropriate amounts of informal feedback from supervisors and colleagues.

Most significant challenges: increased self-awareness of language and communication limitations, and technical challenges.

Most significant preparation for co-op assignments: IEEQ components that facilitate cultural understanding and integration

Benefits of participation in IEEQ: gaining Canadian engineering experience, developing cultural understanding, a licensing pathway in Manitoba: "this system [is a] fast track, not short cut, and is preferred to the traditional route"

"more tolerance and understanding of expectations... and insights [into] cultural differences in the Canadian context"

"the time together connecting with other [immigrants] in this class was very important to encourage and inform each other"

"getting my P.Eng. with APEGM", ... "Canadian work experience", ... "a paper from a Canadian university", ... "going on to Master degree".

"substantially engaged [in engineering work]"

"I used a lot of my background; also, I had to learn new topics"

"it was a little below my technical capabilities but that was perfect for a beginning"

"[I was] quickly accepted in the group, ...excellent teamwork and cooperation"

"writing professional reports and general writing" ... "following the topic in coffee break and lunch", ... "regulations" ... "new kind of design"

"PPEM prepared me to understand the way that my coworkers act and how to respond to determine the situation"

"it helped open the door to the profession by the co-op work term, and helped us get ready for the challenges of the Canadian workplace, and the emphasis on professional licensing" 
Table 4. Summary of post-IEEQ career development

\begin{tabular}{lcc}
\hline Career development parameter & $\begin{array}{c}\text { At nine-month follow-up } \\
\text { questionnaire } \\
(\mathbf{n}=\mathbf{1 2})\end{array}$ & $\begin{array}{c}\text { At 24-month follow-up } \\
\text { questionnaire } \\
(\mathbf{n}=\mathbf{4})\end{array}$ \\
\hline Employment status & & \\
Engineering employment & 9 & 2 \\
Engineering-related employment & 2 & 2 \\
Unemployed & 1 & 0 \\
Self-assessed nature of engineering employment $^{1}$ & & \\
Duties $^{1,2}$, level & $2-3$ & $2-5$ \\
Recommendations $^{1,2}$, level & $2-3$ & $3-5$ \\
Supervision received $^{1,2}$, level & $2-4$ & $3-6$ \\
Leadership authority $^{1,3}$, level & $2-3$ & $3-5$ \\
Other & & \\
Salary range (mean) & $\$ 33,150-\$ 53,700$ & $\$ 34,000-\$ 66,000$ \\
& $(\$ 42,600)$ & $(\$ 55,250)$ \\
Registered as EIT & 12 & 1 \\
Registered as P.Eng. & $\mathrm{n} / \mathrm{a}$ & 3 \\
\hline
\end{tabular}

1. Using the Professional Engineering Classification Rating Guide, excerpted from APEGM annual salary survey tools, and available from www.apegm.mb.ca.

2. Total defined levels on this measure in the Rating Guide range from one to eight. Higher levels correlate with increased responsibilities and independence.

3. Total defined levels on this measure in the Rating Guide range from one to nine. Higher levels correlate with increased coordination, management, and leadership roles.

successful participants. The co-op term is a required program element and is facilitated almost entirely by IEEQ staff. IEEQ has provided Canadian engineering work experience for all participants to date.

Participants perceive IEEQ to be a time-effective alternative to a Confirmatory Exam program, which is congruent with anecdotal observations of IEEQ and APEGM staff. However, to confirm this perception, detailed data on IEG licensing programs going back five years have been requested from APEGM. These data are pending. The social support structure for IEEQ participants addresses the objective of offering a supportive community. These supports were developed in response to participants' experiences with isolation in cohort IEEQ1. These themes were not evident in the data from subsequent cohorts. In contrast, IEEQ2 and IEEQ3 identified support mechanisms within IEEQ (both independently and relative to APEGM Confirmatory Exam programs) as a program strength. Participants derived support from the cohort structure and from interaction with Canadian engineers via guest speakers, industry tours, and Engineers-inResidence. Similarly, findings indicate that participants perceive IEEQ as a vehicle to demonstrate and practice transition and integration into Canadian professional engineering, including technical, cultural, and language aspects. Retrospective perceptions of the program's value focused to a greater degree on cultural integration and understanding and on professional licensing aspects, than on academic gains.

This study is preliminary and exploratory in nature, as no direct parallel engineering programs nor studies were found in the literature. However, the findings support emerging concepts of proposed best practices in recognition and integration programs for immigrant professionals generally, as articulated by the Association of Universities and Colleges of Canada [6] and by the CCPE [1] through the From Consideration to Integration initiative. Proposals for program best-practices include formal recognition of foreign credentials, training in language and communication skills, and work experience, all of which are incorporated into IEEQ. Additionally, collaboration among stakeholders, consideration of participant resources, and strong admission procedures are proposed for best-practice programs and IEEQ has uniquely addressed these areas as well. 


\section{Conclusions}

The objectives of this study were to document and understand the experiences and perceptions of the first three IEEQ cohorts, and to apply these findings to program development and evaluation. Participants identified several strengths of IEEQ, including recognition by APEGM as a licensing pathway, emphasis on cultural understanding, and access to employment. Primary challenges related to the isolation and transitions of adjusting to new systems and environments. In response, IEEQ developed and integrated academic, social, and financial supports into the program. Post-IEEQ, participants have reported career development gains in the nature of their employment, salaries, and progress in meeting licensing requirements. Of significance, the findings confirm that IEEQ is meeting its objectives, validate proposed best practices for FCR programs, and begin to establish an academic knowledge base in this field.

A standard methodological limitation of qualitative research is that the findings represent the views of the voluntary participants and may not represent the views of the entire cohort. The potential effects of self-selection among participants are noted; however, the diversity of responses provides evidence that self-selection did not compromise the findings. The credibility of the findings is further supported by the use of repeated measures (focus group, two questionnaires) to triangulate data.

The study is also limited by the preliminary nature of the research, and findings will evolve as data collection continues through 2008. To continue building a holistic understanding of IEEQ, future work will focus on completing data collection with the first three cohorts, extending data collection to the fourth and fifth cohorts, and augmenting data with one-on-one interviews with other program stakeholders. Future work will also move toward the development of theory, using the constructed understanding of participants' experiences and perceptions in IEEQ toward the development of best practices for qualifications recognition programs for IEGs.

\section{Acknowledgements}

This work was financially supported by University of Manitoba and the Province of Manitoba.

\section{References}

[1] Canadian Council of Professional Engineers, From Consideration to Integration, Final Report from Phase I, Canadian Council of Professional Engineers., Ottawa, ON, 2003.

[2] Statistics Canada, Longitudinal Survey of Immigrants to Canada: Progress and Challenges of New Immigrants in the
Workforce, Report 89-615-XIE, Government of Canada, Ottawa, ON, 2003.

[3] Manitoba Labour and Immigration, Manitoba Immigration Statistics Summary, 2000 Report, Province of Manitoba, Winnipeg, MB, 2001.

[4] Manitoba Finance, Manitoba's Action Strategy for Economic Growth, Province of Manitoba, Winnipeg, MB, 2005.

[5] Human Resources Development Canada, Knowledge Matters: Skills and Learning for Canadians, Report SP482-02-02, Government of Canada, Hull, QC, 2002.

[6] Association of Universities and Colleges of Canada, Mapping Canadian University Capacity, Expertise, and Key Issues Related to Foreign Credential Recognition of Internationally-Educated Professionals, Final Report, Association of Universities and Colleges of Canada., Ottawa, ON, 2006.

[7] M.R. Friesen and M.G. Britton, "Conceptual Framework of the Internationally-Educated Engineers Qualification Pilot Program, University of Manitoba, Canada”, Proceedings of the 2006 ASEE Annual Conference and Exposition, American Society for Engineering Education, Washington, DC, 2006.

[8] M.R. Friesen and M.G. Britton, “Assessment and Evaluation Framework of the Internationally-Educated Engineers Qualification Pilot Program, University of Manitoba, Canada”, Proceedings of the 2006 ASEE Annual Conference and Exposition, American Society for Engineering Education, Washington, DC, 2006.

[9] Bogdan, R.C. and S.K. Biklen, Qualitative Research for Education: An Introduction to Theory and Methods, $3^{\text {rd }}$ edition, Allyn and Bacon, Needham Heights, MA, 1998.

[10] Krueger, R.A, Focus Groups: A Practical Guide for Applied Research, SAGE, Newbury Park, CA, 1988.

[11] Lincoln, Y.S. and E.G. Guba, Naturalistic Inquiry, SAGE, Newbury Park, CA, 1985.

[12] D.J. Unruh, University of Manitoba Access programs, University of Manitoba., Winnipeg, MB, 1992. 